(1)

CrossMark

\title{
Human diaphragm atrophy in amyotrophic lateral sclerosis is not predicted by routine respiratory measures
}

\author{
Raquel Guimarães-Costa ${ }^{1,11}$, Thomas Similowski ${ }^{2,3,11}$, Isabelle Rivals ${ }^{3,4}$, \\ Capucine Morélot-Panzini ${ }^{2,3}$, Marie-Cécile Nierat ${ }^{3}$, Mai Thao Bui ${ }^{5}$, David Akbar ${ }^{6}$, \\ Christian Straus $^{3,7}$, Norma Beatriz Romero ${ }^{5}$, Patrick Pierre Michel $^{6}$, \\ Fabrice Menegaux ${ }^{8}$, François Salachas ${ }^{1}$, Jésus Gonzalez-Bermejo ${ }^{3,9,12}$ and \\ Gaëlle Bruneteau ${ }^{1,10,12}$ on behalf of the RespiStimALS team ${ }^{13}$
}

@ERSpublications

Vital capacity and other widely used respiratory measures are poor predictors of diaphragmatic atrophy. Vital capacity should not be used as the sole selection criteria in clinical trials targeting diaphragm mass or function http://ow.ly/7GEg30mIpCK

Cite this article as: Guimarães-Costa R, Similowski T, Rivals I, et al. Human diaphragm atrophy in amyotrophic lateral sclerosis is not predicted by routine respiratory measures. Eur Respir J 2019; 53: 1801749 [https://doi.org/10.1183/13993003.01749-2018].

ABSTRACT Amyotrophic lateral sclerosis (ALS) patients show progressive respiratory muscle weakness leading to death from respiratory failure. However, there are no data on diaphragm histological changes in ALS patients and how they correlate with routine respiratory measurements.

We collected 39 diaphragm biopsies concomitantly with laparoscopic insertion of intradiaphragmatic electrodes during a randomised controlled trial evaluating early diaphragm pacing in ALS (https:// clinicaltrials.gov; NCT01583088). Myofibre type, size and distribution were evaluated by immunofluorescence microscopy and correlated with spirometry, respiratory muscle strength and phrenic nerve conduction parameters. The relationship between these variables and diaphragm atrophy was assessed using multivariate regression models.

All patients exhibited significant slow- and fast-twitch diaphragmatic atrophy. Vital capacity (VC), maximal inspiratory pressure, sniff nasal inspiratory pressure (SNIP) and twitch transdiaphragmatic pressure did not correlate with the severity of diaphragm atrophy. Inspiratory capacity (IC) correlated modestly with slow-twitch myofibre atrophy. Supine fall in VC correlated weakly with fast-twitch myofibre atrophy. Multivariate analysis showed that IC, SNIP and functional residual capacity were independent predictors of slow-twitch diaphragmatic atrophy, but not fast-twitch atrophy.

Routine respiratory tests are poor predictors of diaphragm structural changes. Improved detection of diaphragm atrophy is essential for clinical practice and for management of trials specifically targeting diaphragm muscle function. 


\section{Introduction}

Amyotrophic lateral sclerosis (ALS) is a progressive neurodegenerative disorder in which motor neuron loss results in extensive paralysis, usually leading to death from respiratory failure approximately 3 years after symptom onset [1]. The progression of ALS-related respiratory muscle weakness can be tracked through sequential measurement of vital capacity (VC) and muscle strength [2], which provide robust information on prognosis [3] and guide the initiation of noninvasive ventilation (NIV) [4]. Within the framework of generalised respiratory muscle weakness, diaphragm involvement plays a major role in ALS-related respiratory insufficiency [5, 6] and is a strong predictor of short-term mortality [7].

Human skeletal muscles are composed of several types of fibre that differ in their myosin heavy chain (MyHC) isoform content. Slow-twitch (Type-1) fibres contain the MyHC-1 isoform and fast-twitch (Type-2) fibres contain the MyHC-2a (Type-2A) or MyHC-2x (Type-2X) isoforms [8]. The normal human diaphragm contains approximately equal amounts of Type-1 and Type- 2 fibres $[9,10]$, accounting for both the extreme endurance of the diaphragm and its ability to support rapid respiratory and postural responses. There have been no histological studies of diaphragm remodelling in ALS patients and therefore it is not known how ALS-related diaphragm changes translate into specific alterations of the results of pulmonary function tests (PFTs).

To address this knowledge gap, we analysed the morphology and composition of muscle fibres in diaphragm biopsies obtained at the time of device implantation from a subset of patients who participated in the RespiStimALS study (https://clinicaltrials.gov; NCT01583088), a randomised controlled trial conducted to test the hypothesis that early diaphragm pacing could delay the need for NIV in ALS patients. The trial was interrupted because of excess mortality in the treatment arm [11, 12]. Here, we performed a comprehensive study of the morphology and composition of diaphragm myofibres and assessed the correlations with routine respiratory tests.

\section{Methods}

\section{Patients}

This study included a subset of the patients from the RespiStimALS study population (www.lppr.org/ RespistimALS/RespiStimALS_Protocol.pdf) [11]. Briefly, the RespiStimALS study enrolled 74 ALS patients over a 34-month period. Inclusion criteria were forced vital capacity (FVC) when sitting of $60-80 \%$ of predicted, age $>18$ years and fulfilment of the revised El Escorial criteria for laboratory-supported probable, clinically probable, or clinically definite ALS [13]. Functional impairment was assessed using the ALS functional rating scale-revised (ALSFRS-R) [14]. All patients underwent laparoscopic insertion of intradiaphragmatic electrodes (NeurRx ${ }^{\oplus}$ DPS, Synapse Biomedical, Cincinnati, OH, USA) and were randomised into active or sham stimulation groups. The patients were asked to participate in a complementary diaphragm biopsy study approved by the appropriate French legal and ethical authority (CPP Ile-de-France VI, Pitié-Salpêtrière, Paris, France; decision number 2012-A00178-35). All patients received detailed information about methods, objectives and risks, and provided written informed consent specific to the biopsy study. A total of 50 patients consented to participate in the biopsy study.

\section{Respiratory measurements}

Preoperative measurements (spirometry, maximal inspiratory pressure (MIP) and maximal sniff nasal inspiratory pressure (SNIP)) were performed at the participating centres upon inclusion and then again at

Affiliations: ${ }^{1}$ AP-HP, Groupe Hospitalier Pitié-Salpêtrière Charles Foix, Département de Neurologie, Centre référent SLA, Paris, France. ${ }^{2} \mathrm{AP}-\mathrm{HP}$, Groupe Hospitalier Pitié-Salpêtrière Charles Foix, Service de Pneumologie, Médecine Intensive et Réanimation, Département R3S, Paris, France. ${ }^{3}$ Sorbonne Université, INSERM, UMRS1158 Neurophysiologie Respiratoire Expérimentale et Clinique, Paris, France. ${ }^{4}$ Equipe de Statistique Appliquée, ESPCI Paris, PSL Research University and Sorbonne Université, INSERM, UMRS1158 Neurophysiologie Respiratoire Expérimentale et Clinique, Paris, France. ${ }^{5}$ AP-HP, Groupe Hospitalier PitiéSalpêtrière Charles Foix, Unité de Morphologie Neuromusculaire, Institut de Myologie, Paris, France. ${ }^{6}$ CELIS Cell Culture Core Facility, Institut du Cerveau et de la Moelle Épinière, ICM, INSERM U1127, CNRS UMR 7225, Sorbonne Université, Paris, France. ${ }^{7}$ AP-HP, Groupe Hospitalier Pitié-Salpêtrière Charles Foix, Service des Explorations de la Fonction Respiratoire, de l'Exercice et de la Dyspnée, Département R3S, Paris, France. ${ }^{8} \mathrm{AP}-\mathrm{HP}$, Groupe Hospitalier Pitié-Salpêtrière Charles Foix, Département de Chirurgie Générale et Endocrinologique, Paris, France. ${ }^{9}$ AP-HP, Groupe Hospitalier Pitié-Salpêtrière Charles Foix, Unité Fonctionnelle SSR Respiratoire, Service de Pneumologie, Médecine Intensive et Réanimation Département R3S, Paris, France. ${ }^{10}$ Institut du Cerveau et de la Moelle Épinière, ICM, INSERM U1127, CNRS UMR 7225, Sorbonne Université, Paris, France. ${ }^{11}$ Joint first authors. ${ }^{12}$ Joint last authors. ${ }^{13} \mathrm{~A}$ list of contributors to the RespiStimALS study can be found at the end of this article.

Correspondence: Gaëlle Bruneteau, Dépt de Neurologie, Centre Référent SLA, Institut du Cerveau et de la Moelle Épinière, ICM, Hôpital Pitié-Salpêtrière, 47-83 Bd de l'Hôpital, 75013 Paris, France. E-mail: gaelle. bruneteau.aphplagmail.com 
the coordinating centres before surgery (delay before surgery: median 1 day (range 1-19 days); interval between enrolment and preoperative measures: median 16 days (0-78 days)). The analysis reported here used only the values obtained from preoperative measurements. Diagnostic phrenic stimulation was also performed [11], which provided values for twitch transdiaphragmatic pressure (defined as the difference between intrathoracic pressure and intra-abdominal pressure) and the latency and amplitude of the diaphragm compound motor action potentials (CMAPs).

\section{Morphometric analysis of diaphragm muscle samples}

Diaphragm biopsy samples were collected at the end of the surgical procedure as described [15]. Samples (approximately $8 \times 2.5 \mathrm{~mm}$ ) were removed from the left costal diaphragm adjacent to its insertion on the tenth rib, rapidly frozen in isopentane precooled with liquid nitrogen and stored at $-80{ }^{\circ} \mathrm{C}$ until analysis of muscle fibre type and distribution by indirect immunofluorescence.

In brief, 8 - $\mu \mathrm{m}$-thick cryostat sections were blocked with IgG-free 3\% bovine serum albumin (BSA) for $1 \mathrm{~h}$ at room temperature while protected from light. The sections were then washed and incubated with antibodies to MyHC-1 (BA-D5-s; Developmental Studies Hybridoma Bank (DSHB), University of Iowa, IA, USA) or MyHC-2a (SC-71-s; DSHB) for $2 \mathrm{~h}$ at room temperature while protected from light. After washing, the sections were incubated with an antibody to MyHC-2x (6H1-s; DSHB) overnight at $4{ }^{\circ} \mathrm{C}$. The sections were then incubated with the appropriate secondary antibody (CY3-conjugated goat anti-mouse IgG2b (1:100; Interchim \#115-165-207), Alexa Fluor 488-conjugated goat anti-mouse IgG1 (1:100; Interchim \#115-545-205), or AMCA-conjugated goat $\mathrm{F}\left(\mathrm{ab}^{\prime}\right) 2$ anti-mouse $\operatorname{IgM}$ (1:50; Interchim \#115-156-020)) (all from Interchim, Monluçon, France) for $1 \mathrm{~h}$ at room temperature, washed and analysed. A minimum of 400 muscle fibres were analysed for each patient.

Quantitative analysis of muscle histology was performed using an ArrayScan XTI automated workstation equipped with HCS Studio 3.0 software (Thermo Scientific, Courtaboeuf, France). Single images were acquired at $10 \times$ objective and at different wavelengths to generate merged composite images of tissue sections. Single wavelength composite images were processed for quantitative analysis using the Morpho algorithm to allow automated segmentation of regions of interest. Fibres lying only partially within the picture frame were automatically excluded. The software also included built-in image editing to manually inspect and adjust fibre boundaries when required. Muscle fibre size was estimated by measuring the minimum diameter of each fibre. As the RespiStimALS study did not include "normal subjects", we based our cut-offs for atrophy on the reference data formerly obtained in subjects without neuromuscular disorders and with normal respiratory function by the Paris Respiratory Muscle Group [10]. For each fibre type, atrophic fibres were defined as myofibres with a diameter two standard deviations (SD) below the mean diameter of the same diaphragm muscle fibre type [10]. The cut-off diameters for atrophic Type-1 and Type-2 fibres were set at 54.02 and $47.08 \mu \mathrm{m}$, respectively. In normal muscle, fibres innervated by different motor neurons are intermingled, leading to an apparently random mix of Type-1 and Type-2 fibres. Fibre-type grouping (i.e. grouping of muscle fibres of the same type) is a well-established indicator of partial denervation followed by reinnervation [16]. Fibre-type grouping can be assessed by determining the number of fibres surrounded on all sides by fibres of the same type (i.e. enclosed fibres) [17]. To be considered as enclosed, one muscle fibre must be exclusively in contact with fibres of the same type. To quantify fibre-type grouping we manually determined the number of muscle fibres contained in each cluster of enclosed fibres in each muscle section. All morphometric analyses were performed by an investigator blinded to the patient's stimulation group. For each muscle sample, fibre-type grouping and size were derived from analysis of 400-6000 fibres (mean 2037).

\section{Statistical analysis}

Statistical analyses were conducted with XLSTAT 2017 (Addinsoft, Paris, France) and MATLAB (Statistics and Machine Learning Toolbox 11.2, R2017b, MathWorks, Natick, MA, USA). Welch's tests were used to compare the mean minimal diameter of muscle fibres and fibre-type proportions between ALS patients and historical controls. Other continuous variables were compared with the Mann-Whitney U-test, categorical data were analysed with Fisher's exact test and correlations between variables were analysed using Spearman's nonparametric rank correlation test. Multivariate analyses were performed to evaluate the relationship between respiratory data and muscle fibre atrophy. Variables yielding a Spearman correlation p-value of less than 0.3 were entered into generalised linear models. The response variables being proportions (of atrophic fibres), both logistic and linear functions of a full two degree polynomial were considered. The final models were determined using a forward or backward stepwise regression. All statistics were two-tailed and the level of significance was set at 0.05 .

\section{Results}

Of the 74 patients enrolled in RespiStimALS, 50 agreed to participate in the biopsy study. Of the 50 biopsies, 11 were of insufficient quality for analysis (samples were too small and/or consisted mainly of 
fibrovascular and adipose tissue). The demographic and clinical characteristics of the remaining 39 patients (active stimulation $(n=19)$, sham stimulation $(n=20)$ ) are described in table 1 .

Representative micrographs of the specimens from an ALS patient, featuring the characteristic diaphragm changes, are shown in figure 1. Numerous small-caliber angular Type-1 and Type-2 fibres are evident, with some grouping of the same fibre types (figure 1a). In some patients, such fibre-type grouping leads to a predominance of a single fibre type (figure $1 \mathrm{~b}$ ). The results in the whole study population are summarised in table 2 (mean, median (interquartile range (IQR)) and figure 2 (median (range))). The median proportion of Type-1 muscle fibres was 55.5\%, albeit with striking variability (range 30.1-94.1; figure 2a). The mean diameters for Type-1 and Type-2 fibres were significantly smaller for ALS patients (54.2 $\mu \mathrm{m}$ and $44.9 \mu \mathrm{m}$, respectively) than for normal subjects $(59.7 \mu \mathrm{m}$ and $55.3 \mu \mathrm{m}$, respectively [10]), reflecting the marked atrophy of both fibre types in the patient population (table 2 and figure $2 b$ ). Instead of the checkerboard pattern of Type- 1 and Type- 2 fibres typical of a normal diaphragm, "enclosed" muscle fibres that were only in contact with fibres of the same type (known as "fibre-type grouping") were evident in most of the ALS patient sections. The median proportion of enclosed Type-1 fibres was significantly higher than that of enclosed Type-2 fibres (5.3\% (range 0-35.6) versus $1.0 \%$ (range 0-12.5), $\mathrm{p}<0.01$; table 2

TABLE 1 Demographic and clinical characteristics of amyotrophic lateral sclerosis (ALS) patients who underwent diaphragm biopsy and normal patients

\begin{tabular}{|c|c|c|}
\hline Characteristic & ALS patients $(n=39)$ & Normal patients $(n=10)[10]$ \\
\hline Age years & $56 \pm 12.4(47-63)$ & $47 \pm 4.4$ \\
\hline Male & 23 & - \\
\hline Bulbar onset & $7(18)$ & - \\
\hline Disease duration" months & $25(18-36)$ & - \\
\hline ALSFRS-R score ${ }^{\#}$ & $33(26-38)$ & - \\
\hline ALSFRS-R respiratory subscore ${ }^{\#, ~ ๆ ~}$ & $11(10-12)$ & - \\
\hline Riluzole treatment & $39(100)$ & - \\
\hline Active stimulation group & 19 & - \\
\hline Deceased at last follow-up & 24 & - \\
\hline Disease duration months ${ }^{+}$ & $53(39-70)$ & - \\
\hline \multicolumn{3}{|l|}{ Pulmonary function tests } \\
\hline \multicolumn{3}{|l|}{ Sitting } \\
\hline FVC \% predicted & $83 \pm 14.4(75-97)$ & $108 \pm 4.8$ \\
\hline SVC \% predicted & $79(73-94)$ & - \\
\hline FRC $\%$ predicted & $92 \pm 18.4(78-104)$ & $106 \pm 5.4$ \\
\hline IC L & $2.3(1.8-2.7)$ & - \\
\hline MIP \% predicted & $73(50-82)$ & - \\
\hline SNIP \% predicted & $57(47-65)$ & - \\
\hline \multicolumn{3}{|l|}{ Supine } \\
\hline SVC $\%$ predicted & $78(71-87)$ & - \\
\hline FRC $\%$ predicted & $72(60-80)$ & - \\
\hline IC L & $2.5(1.9-3)$ & - \\
\hline Twitch transdiaphragmatic pressure ${ }^{\S} \mathrm{cm} \mathrm{H}_{2} \mathrm{O}$ & $19.8(11.4-25.4)$ & - \\
\hline Left phrenic nerve CMAP amplitude ${ }^{f} \mu \mathrm{V}$ & $497(374.7-684.3)$ & - \\
\hline Left phrenic nerve terminal latency ${ }^{f} \mathrm{~ms}$ & $7.8(7.1-8.2)$ & - \\
\hline
\end{tabular}

Data are presented as $n, n(\%)$, median (interquartile range (IQR)), or median $\pm S D(I Q R)$. IQR data is not available in the reference data formerly obtained in normal patients by the Paris Respiratory Muscle Group and as such we present standard deviation for comparison [10]. For sitting slow vital capacity (SVC), functional residual capacity (FRC) and inspiratory capacity (IC), n=38; for maximal inspiratory pressure (MIP), $n=38$; and for sniff nasal inspiratory pressure (SNIP), $n=35$. For supine SVC, $n=37$. ALSFRS-R: ALS functional rating scale-revised (score: 0 (total disability) to 48 (no disability)); FVC: forced vital capacity; CMAP: compound motor action potential. " : at the time of biopsy. ": the ALSFRS-R respiratory subscore (scale: 0 to 12) includes the three items from the ALSFRS-R that evaluate respiratory symptoms (dyspnoea, orthopnoea and use of mechanical ventilation). ${ }^{+}$: disease duration is the time between first ALS symptoms and death or last follow-up. $\S_{\text {: }}$ twitch transdiaphragmatic pressure is the highest value obtained from cervical magnetic stimulation or bilateral anterior magnetic stimulation $(n=31)$. Phrenic stimulation was performed according to the American Thoracic Society/European Respiratory Society guidelines [18]. $f_{\text {: }}$ values are from the left side only to match the diaphragm biopsy location. With the exception of a significant over-representation of women in the sham group (male/female ratio 7:13) versus the active stimulation group (male/female ratio 16:3; $\mathrm{p}=0.003$ ), there were no significant differences between patients in the active and sham groups. 

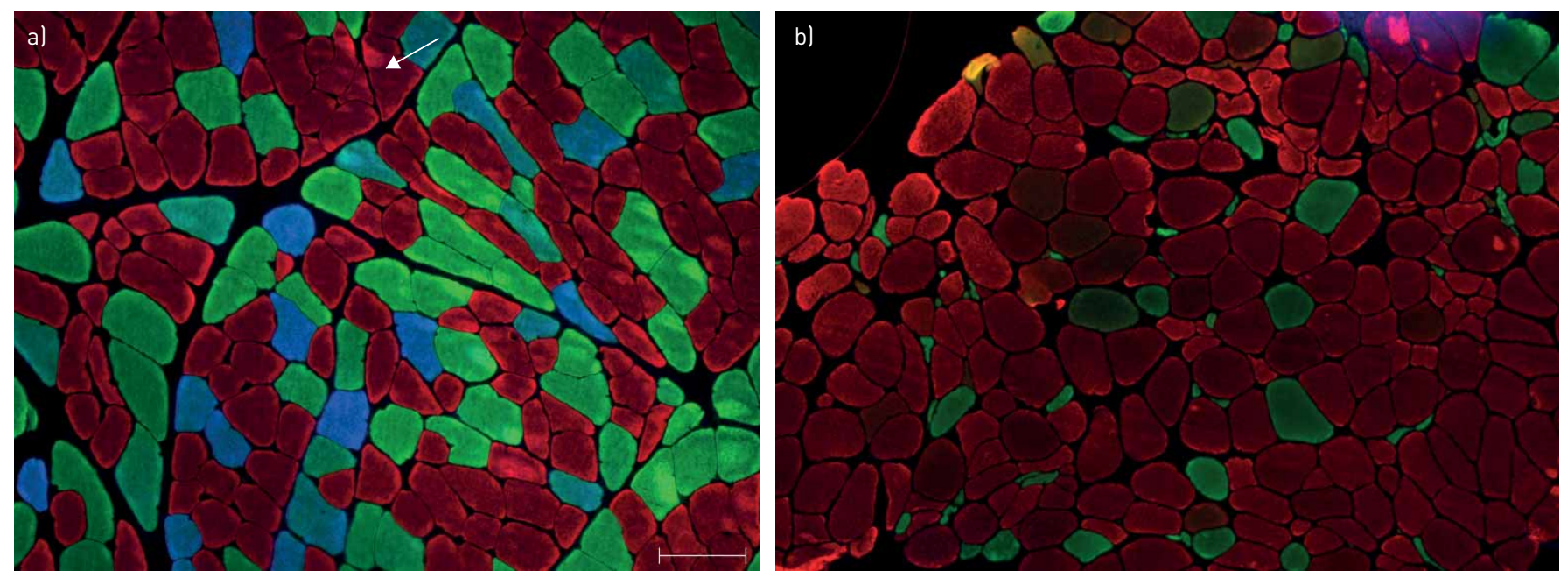

FIGURE 1 Diaphragm remodelling in amyotrophic lateral sclerosis (ALS) patients with representative immunofluorescent staining of MyHC I (Type-1 slow-twitch fibres, red), MyHC Ila (Type-2 fast-twitch fibres, green) and MyHC IIx (Type-2 fast-twitch fibres, blue) in diaphragm muscle cross-sections from ALS patients observed under a light microscope ( $\times 16$ objective). a) Numerous small-calibre angular Type-1 and Type-2 fibres can be seen, together with enclosed fibres (fibres surrounded on all sides by fibres of the same type; arrow; scale bar=100 $\mu \mathrm{m}$ ). b) In some patients, fibre-type grouping affects predominantly Type-1 muscle fibres, leading to Type-1 predominance.

and figure 2c). There was a positive correlation between disease duration at the time of biopsy and the proportion of enclosed Type- 1 muscle fibres (Spearman's rank correlation coefficient ( $r)=0.337$ ( $95 \%$ CI 0.015-0.596), $\mathrm{p}=0.036$ ). However, no correlations were detected between histological characteristics and age, ALSFRS-R score, or respiratory ALSFRS-R subscore at the time of biopsy. Notably, there were no significant differences between the active and sham stimulation groups with respect to fibre typing, fibre atrophy, or fibre-type grouping (table 3).

Significant correlations were detected between several respiratory parameters and muscle morphometric measures in the combined active and sham groups $(n=39)$. Sitting FVC correlated modestly with the proportion of enclosed Type- 1 muscle fibres ( $r=0.317$ (95\% CI -0.006-0.581), $p=0.049$; figure 3a). The reduction in slow vital capacity (SVC) in the supine posture $(\triangle \mathrm{SVC})$ correlated weakly with the proportion of Type- 2 atrophic myofibres $(\mathrm{r}=0.325$ (95\% CI $-0.007-0.593), \mathrm{p}=0.05)$. There were no other correlations between sitting or supine SVC and their difference and muscle histological variables. Similarly, MIP, SNIP

TABLE 2 Histological characteristics of the diaphragm in amyotrophic lateral sclerosis (ALS) patients

\begin{tabular}{|c|c|c|c|c|c|c|c|}
\hline \multirow[t]{2}{*}{ Characteristic } & \multicolumn{4}{|c|}{ ALS patients } & \multicolumn{2}{|c|}{$\begin{array}{c}\text { Normal } \\
\text { patients } \\
\text { [10] }\end{array}$} & \multirow[t]{2}{*}{ p-value } \\
\hline & Mean & Median & SD & IQR & Mean & SD & \\
\hline \multicolumn{8}{|l|}{ Type-1 fibres } \\
\hline Minimum diameter $\mu \mathrm{m}$ & 54.2 & 53.4 & 8.4 & $48.9-59.9$ & 59.7 & 0.9 & 0.00026 \\
\hline Proportion \% & 54.7 & 55.5 & 14.6 & $42.5-65.1$ & 53.2 & 1.8 & NS \\
\hline Proportion of atrophic fibres $\#$ & 56.0 & 57.1 & 15.5 & $46.3-67.3$ & NA & - & - \\
\hline Proportion of enclosed fibres ${ }^{\pi} \%$ & 8.3 & 5.3 & 8.3 & $3.2-11.2$ & NA & - & - \\
\hline \multicolumn{8}{|l|}{ Type-2 fibres } \\
\hline Minimum diameter $\mu \mathrm{m}$ & 44.9 & 44.3 & 14.8 & $34.1-54.2$ & 55.3 & 1.3 & 0.000098 \\
\hline Proportion \% & 45.3 & 44.5 & 14.6 & $34.9-57.6$ & 46.8 & 1.8 & NS \\
\hline Proportion of atrophic fibres ${ }^{\#} \%$ & 56.0 & 53.7 & 24.5 & $39.4-74.5$ & NA & - & - \\
\hline Proportion of enclosed fibres $\%$ & 1.9 & 1.0 & 2.6 & $0.4-2.3$ & NA & - & - \\
\hline
\end{tabular}

IQR: interquartile range; NA: not applicable; NS: nonsignificant. " : atrophic fibres are those with a diameter of two standard deviations below the mean diameter measured in normal patients. In the absence of more recent normative data, we based our cut-offs for atrophy on the reference data formerly obtained by the Paris Respiratory Muscle Group [10]. As IQR data is not available in these reference data, we present standard deviation for comparison; ๆ : enclosed muscle fibres are those surrounded on all sides by fibres of the same type [17]. 


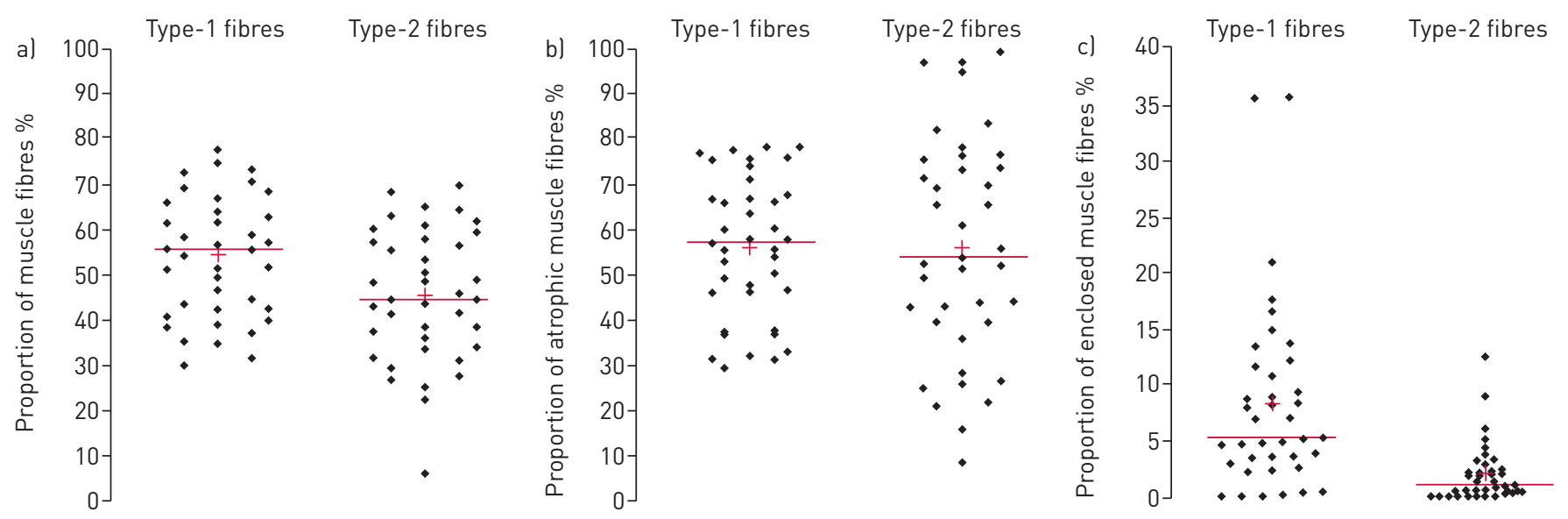

FIGURE 2 Heterogeneity of diaphragm histology in amyotrophic lateral sclerosis (ALS) patients (n=39). Scattergram representations are as follows: a) percentage of total Type- 1 and Type-2 fibres; b) percentage of atrophic Type- 1 and Type-2 fibres; and c) percentage of enclosed Type- 1 and Type-2 fibres. Each dot represents one patient. Median (range) values are: a) 55.5\% (30.1-94.1\%) for Type-1 and 44.5\% (5.9-69.9\%) for Type-2 fibres; b) 57.1\% (29.4-78.2\%) for Type-1 and 53.7 (8.6-99.2\%) for Type-2 fibres; and c) 5.3\% (0-35.6\%) for Type-1 and 1.0\% (0-12.5\%) for Type-2 fibres $(p<0.01)$. A red cross indicates the mean value and a red line indicates the median value.

and twitch transdiaphragmatic pressure values did not correlate with any diaphragm histological characteristics. Inspiratory capacity (IC) was the respiratory variable most clearly correlated with histological characteristics of the diaphragm, although these correlations were generally modest $(\mathrm{r}<0.4)$. Sitting IC correlated positively with Type- 1 muscle fibre diameter ( $\mathrm{r}=0.380$ (95\% CI 0.062-0.628), $\mathrm{p}=0.017$; figure $3 \mathrm{~b}$ ) and negatively with the proportion of Type- 1 atrophic fibres ( $\mathrm{r}=-0.380$ (95\% CI -0.628 to $-0.062), p=0.017$; figure $3 \mathrm{c}$ ). Supine IC correlated negatively with the proportion of Type- 1 muscle fibres ( $\mathrm{r}=-0.336$ (95\% CI -0.598 to -0.009 ), $\mathrm{p}=0.039$; figure $3 \mathrm{~d}$ ), reflecting the fibre-type composition and positively with the proportion of enclosed Type- 2 muscle fibres $(r=0.356$ (95\% CI 0.030-0.613), $\mathrm{p}=0.029$; figure 3e), reflecting the reinnervation of fast-twitch myofibres. Finally, sitting functional residual capacity (FRC) correlated negatively with the proportion of Type- 1 muscle fibres ( $r=-0.365$ (95\% CI -0.620 to $-0.040), p=0.025$; figure 3f).

With respect to electrophysiology, a modest positive correlation was observed between the amplitude of the left phrenic nerve CMAP and the proportion of Type- 2 muscle fibres ( $r=0.323$ (95\% CI 0.000-0.585), $\mathrm{p}=0.045$ ), whereas terminal latency did not correlate with any diaphragm histological characteristics.

Multivariate analysis showed that sitting FRC, supine FRC, sitting IC, supine IC and SNIP yielded a correlation with the proportion of atrophic Type-1 fibres $(\mathrm{p}<0.3)$, whereas only sitting SVC and $\Delta$ SVC correlated with the proportion of atrophic Type-2 fibres (see the supplementary material (supplementary figure S1) for the correlation map). The linear models outperformed the logistic models and the results of a backward or forward stepwise linear regression analysis on the full second degree polynomial model

TABLE 3 Comparison of histological characteristics of the diaphragm in the active and sham stimulation groups

\begin{tabular}{|c|c|c|c|}
\hline \multirow[t]{2}{*}{ Characteristic } & \multicolumn{2}{|c|}{ Stimulation group } & \multirow[t]{2}{*}{ p-value } \\
\hline & Active & Sham & \\
\hline \multicolumn{4}{|l|}{ Type-1 fibres } \\
\hline Minimum diameter $\mu \mathrm{m}$ & $53.7(51.0-60.3)$ & $50.9(46.9-59.0)$ & NS \\
\hline Proportion \% & $54.5(41.5-61.5)$ & $57.3(44.5-67.2)$ & NS \\
\hline Proportion of atrophic fibres ${ }^{\#} \%$ & $55.6(46.5-60.1)$ & $64.8(44.1-75.4)$ & NS \\
\hline Proportion of enclosed fibres $\%$ & $5.1(3.6-11.9)$ & $6.1(2.5-9.0)$ & NS \\
\hline \multicolumn{4}{|l|}{ Type-2 fibres } \\
\hline Minimum diameter $\mu \mathrm{m}$ & $45.0(38.6-55.4)$ & $40.8(33.6-53.7)$ & NS \\
\hline Proportion of atrophic fibres ${ }^{\#} \%$ & $52.0(37.6-69.3)$ & $67.4(41.9-76.3)$ & NS \\
\hline Proportion of enclosed fibres $\%$ & $1.3(0.5-2.5)$ & $0.7(0.3-2.1)$ & NS \\
\hline
\end{tabular}



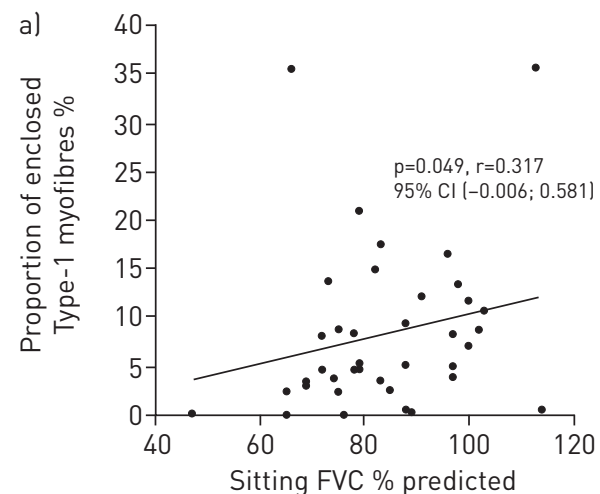

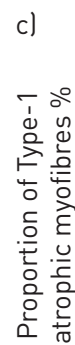

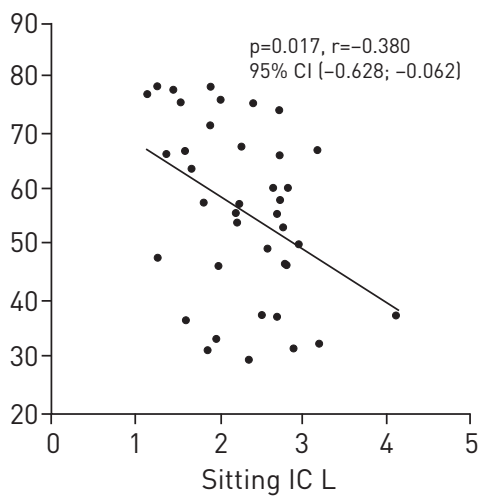

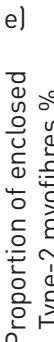

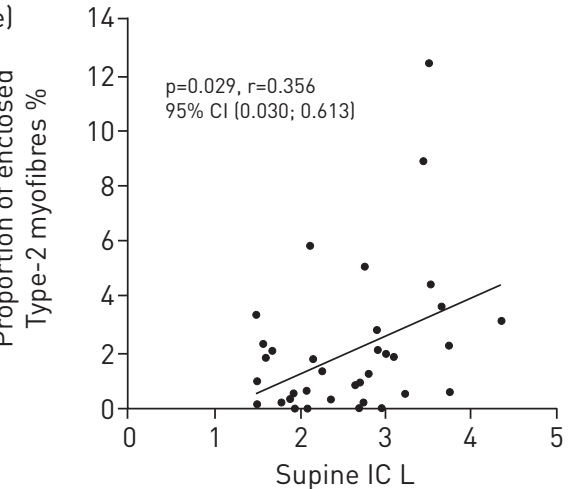

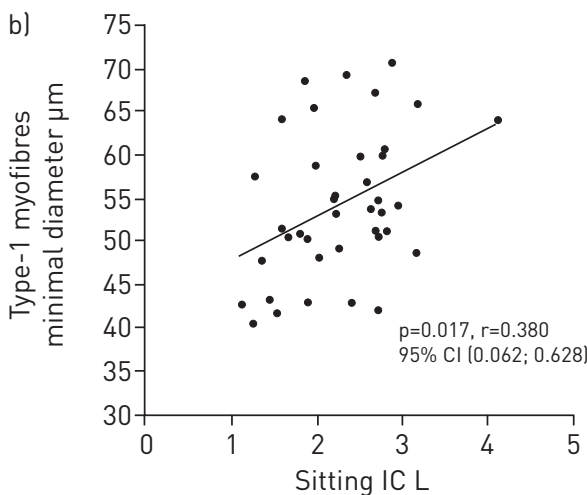
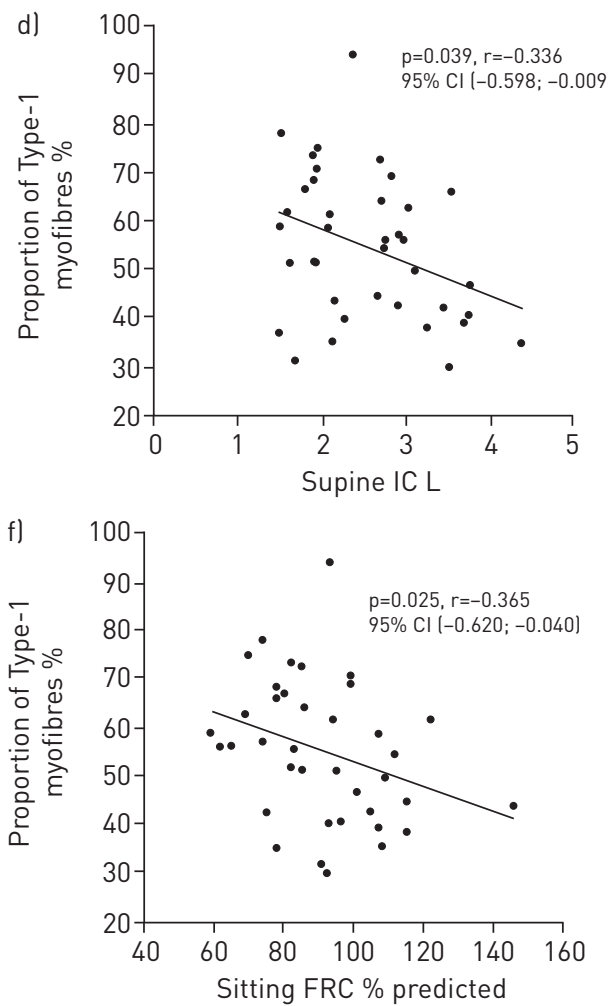

FIGURE 3 Relationship between pulmonary function tests and diaphragm histological properties. a) Positive correlation between sitting forced vital capacity (FVC) and percentage of enclosed Type-1 (slow-twitch) fibres (Spearman's rank correlation coefficient $(r)=0.317)$. b) Positive correlation between sitting inspiratory capacity (IC) and Type-1 fibre diameter ( $r=0.380)$. c) Negative correlation between sitting IC and percentage of atrophic Type-1 fibres ( $r=-0.380)$. d) Negative correlation between supine IC and percentage of Type-1 fibres ( $r=$ -0.336). el Positive correlation between supine IC and percentage of enclosed Type-2 (fast-twitch) fibres, where enclosed muscle fibres are those surrounded on all sides by fibres of the same type li.e. fibre-type grouping indicating partial denervation followed by reinnervation) ( $r=0.356)$. f) Negative correlation between sitting functional residual capacity (FRC) and percentage of Type-1 fibres $(r=-0.365)$.

were identical. The characteristics of the final model for predicting Type-1 atrophy are shown in table 4 . Multiple linear regression showed that increased Type-1 myofibre atrophy was independently associated with higher sitting FRC (regression coefficient 0.0041, p=0.00046), lower sitting IC (regression coefficient $-0.0008, \mathrm{p}=0.01$ ) and lower SNIP (regression coefficient $-0.0031, \mathrm{p}=0.0031$ ). Overall, $48.33 \%$ of the variance of diaphragm slow-twitch atrophy could be explained by the model. In contrast, no model gave a significantly better prediction of Type- 2 myofibre atrophy than a constant model.

\section{Discussion}

This study provides the first description of structural changes in the diaphragm of ALS patients. We detected profound diaphragm anomalies in patients who were considered to have only mild respiratory abnormalities based on VC measurements. 
TABLE 4 Multivariate analysis of the relationship between respiratory parameters and the proportion of atrophic Type-1 fibres

\begin{tabular}{lcc} 
Independent variable & Regression coefficient & p-value \\
\hline Sitting FRC \% predicted & 0.0041 & 0.00046 \\
Sitting IC \% predicted & -0.00078 & 0.01 \\
SNIP \% predicted & -0.0031 & 0.0031 \\
All versus constant model & - & 0.000043
\end{tabular}

FRC: functional residual capacity; IC: inspiratory capacity; SNIP: sniff nasal inspiratory pressure.

These histological diaphragm modifications included morphological changes associated with denervation consequent to motor neuron death, with significant atrophy of both slow-twitch and fast-twitch muscle fibres. Interestingly, in this supposedly homogeneous patient population, there was a striking degree of inter-patient variability, with some specimens exhibiting $>90 \%$ atrophic fast-twitch myofibres. Significant fibre-type grouping was also observed, mainly involving Type-1 fibres. In the context of chronic denervation, fibre-type grouping is a consequence of collateral reinnervation of denervated muscle fibres, with the degree of grouping reflecting the extent of reinnervation [19]. Since motor neuron impulse patterns determine myofibre $\mathrm{MyHC}$ expression [20], fibre-type grouping results from the transformation of Type-1 to Type-2 fibres, or vice versa, under the influence of reinnervating collateral sprouts [19]. Denervated myofibres can be reinnervated by both slow-firing (Type-1) and fast-firing (Type-2) motor neurons. Nevertheless, as we observed in our ALS patients, a preponderance of Type-1 grouping and an increase in the proportion of muscle area occupied by Type-1 fibres are common after reinnervation, possibly because Type-1 motor neurons may have a faster axonal growth rate [21]. As for muscle fibre atrophy, we observed a highly variable degree of fibre-type grouping amongst our patients. This reflects individual disparities in the efficiency of reinnervation, a higher proportion of reinnervated fibres being associated with longer disease duration [22]. Reinnervation of denervated muscle fibres by the remaining motor neurons is a crucial compensatory process in ALS [23-25]. In limb muscles, motor function is preserved as long as motor neuron loss is compensated for by reinnervation [22]. Our results in the diaphragm are in line with these data, a higher reinnervation of slow-twitch myofibres being associated with a higher sitting FVC. We did not detect any correlation between reinnervation of fast-twitch myofibres and FVC, which may be explained by the fact that reinnervated fibres were predominantly slow-twitch rather than fast-twitch myofibres. However, we found that a higher reinnervation of fast-twitch myofibres was associated with a higher supine IC.

Our analysis uncovered a striking contrast between the histological evidence of advanced diaphragm atrophy and the moderate impairment of VC that defined our study population (as dictated by the study design). This is not surprising as inspiratory neck muscles have been reported to compensate for diaphragm dysfunction to preserve VC in ALS patients [26]. Similarly, diaphragm atrophy did not individually correlate with MIP or SNIP, which are global inspiratory muscle tests [27]. These tests are undoubtedly useful to evaluate inspiratory muscle function decline and as biomarkers of survival in ALS [2], but our study shows that they should not be used as markers of diaphragm structural impairment. We found that IC was the only respiratory variable to correlate significantly with both diaphragm atrophy (where high sitting IC is associated with a lower proportion of Type-1 atrophic myofibres) and reinnervation (where high supine IC is associated with a higher proportion of enclosed Type-2 myofibres). This novel finding is of particular interest because, in contrast to VC, IC involves the diaphragm and extradiaphragmatic inspiratory muscles, but not the expiratory muscles.

Our finding that FRC, a static lung volume determined solely by the equilibrium between inward lung recoil and outward chest wall recoil, correlates with a diaphragmatic structural characteristic (lower percentage of Type-1 fibres) is somewhat surprising. However, the diaphragm is a component of the chest wall, the trophicity of which influences lung volume and compliance [28]. ALS-related diaphragm remodelling could influence FRC through changes in its mechanical properties. Indeed, it has been shown that, during muscle remodelling, modifications in fibre-type composition were accompanied by changes in the relative proportions of muscle collagen major forms, with transformation of muscle fibres from fast to slow being associated with an increase in Type-1 collagen content [29]. Predominantly "slow" muscles also contain more abundant and cross-linked collagen, which can increase muscle rigidity [30]. In our study, a lower FRC was associated with a higher proportion of Type-1 myofibres in the diaphragm, suggesting that a predominantly "slow-twitch diaphragm" produces a lower outward elastic recoil. This may thus be due to modifications in diaphragm collagen composition concomitant to slow-fibre enrichment. 
There have been few studies relating muscle morphometric changes to motor function in ALS. In a study of 24 patients with motor neuron disease, including 21 ALS cases, PATTEN et al. [31] found that the density of atrophic myofibres (the number of atrophic fibres per $\mathrm{cm}^{2}$ ) correlated with the degree of muscle weakness. However, this study is difficult to interpret because the site of the biopsy sample for each patient was not specified. In a study by FroEs et al. [32], performed in 19 patients with motor neuron disease (including six ALS patients), pathological features of denervation and reinnervation with considerable variation between patients (as in our study in the diaphragm) were observed in the quadriceps rectus femoris muscle. Also, as in our study, there was no correlation between quadriceps morphometry and its motor function (i.e. knee extension). However, knee extension involves not only the quadriceps rectus femoris (the only muscle biopsied), but also the vastus lateralis, vastus medialis and vastus intermedius muscles. We thus believe that the lack of strong correlation between diaphragm histology and lung volumes or respiratory pressures in our study is probably not an intrinsic feature of ALS. Rather, it probably illustrates that it is unlikely to find strong correlations between the morphometric features of one muscle (e.g. the diaphragm) and the strength of a group of muscles (i.e. those involved in lung volumes).

In ALS, the reduction of diaphragm CMAP amplitude has prognostic value [33], but a dramatically reduced CMAP can also coexist with VC values within the normal range [34]. In our study, a smaller CMAP amplitude was associated with a lower proportion of fast-twitch myofibres, suggesting that M-wave amplitude is mainly reduced by loss of these fibres in ALS patients. This is consistent with early selective loss of the largest and fastest motor units, as previously reported in several ALS mouse models [24, 25]. However, the size or reinnervation of Type-2 fibres did not correlate with CMAP amplitude, indicating that diaphragm remodelling is only partially reflected by changes in this parameter. Although other studies have suggested that prolongation of phrenic nerve terminal latency may be an early indicator of respiratory involvement in ALS [35], we did not detect any association between terminal latency and diaphragm histology. We also saw no association between twitch transdiaphragmatic pressure and muscle atrophy. This is not particularly surprising in so far as twitch transdiaphragmatic pressure depends not only on myofibre size [36] and fibre-type composition [37], but also on other factors like lung volume (which influences diaphragm length) or mechanical properties of the abdominal and thoracic wall [18].

Our inability to detect a correlation between standard respiratory variables and diaphragm atrophy does not diminish the value of these variables for assessing global respiratory muscle strength [2], predicting prognosis [3] and guiding the indications for NIV in ALS [4]. However, it does indicate that these indices cannot be used to predict diaphragm structural changes (in particular myofibre atrophy). Instead, we suggest that a combination of sitting FRC, sitting IC and SNIP are better indicators of atrophy. Specifically, a low FRC with preserved sitting IC and SNIP is suggestive of a low proportion of Type-1 atrophic muscle fibres and a moderate degree of diaphragm atrophy. Notably, we did not identify a model able to predict fast-twitch myofibre atrophy.

Our study has limitations. First, the RespiStimALS trial, by its nature, only involved ALS patients. It was therefore impossible to compare our diaphragm biopsy data with true control data (namely data obtained at the same time with the same procedure). To determine the cut-off value for atrophic fibres, we used historical data obtained during surgical procedures in ten patients with normal lung function [10]. In this previous study, healthy subjects were slightly younger (mean age 47.4 years) and fibre type was assessed by the myosine ATPase histochemical methodology [38]. However, a good correlation has been shown between histochemically determined fibre types and MyHC content in human muscle, both for single fibres [39] and entire biopsy specimens [40]. Furthermore, in another study of the human diaphragm in patients with chronic obstructive pulmonary disease (COPD) [41], no differences were found with respect to fibre type proportion when comparing the ATPase method and the immunocytochemical determination of MyHC isoform content used here. We therefore believe that this issue does not negate the impact of our findings. Another limitation of the study is that, for obvious reasons, we could only examine diaphragm samples at one time point. Serial sampling during follow-up would have improved our understanding of the mechanisms underlying the adverse effects of diaphragm pacing. It is important to note, however, that we observed no differences in diaphragm histology between patients in the active stimulation and sham stimulation groups. Therefore, the results of the RespiStimALS trial cannot be explained by differences in diaphragm atrophy and remodelling between patients in these groups. Finally, it should be noted that the pulmonary function values used in this study (table 1) differ in part from those in the RespiStimALS main report [11]. This is because here we present preoperative values collected at the study coordinating centre, while the main report gives inclusion values collected at the various recruiting centres. This probably accounts for the median VC in our 39 patient biopsy subpopulation which falls outside the $60-80 \%$ predicted window that was an inclusion criterion for RespiStimALS. Several explanations can be considered for this difference, which illustrate the difficulty and variability of FVC measurement in ALS patients. While some patients may have deteriorated between the two measurements, 
others probably produced better FVC values during the second set of measurements because of a learning effect, enhanced motivation due to the prospect of inclusion in the trial, or more active coaching from highly trained and specialised laboratory technicians.

\section{Conclusion}

Our findings indicate that severe histological alterations in the diaphragm, as indicated by muscle atrophy and compensatory (reinnervation-induced) remodelling, are already present in many ALS patients who are considered to have only modest respiratory involvement. We posit that the respiratory measurements routinely used to monitor ALS patients (mainly VC and respiratory pressures) are inadequate for diagnosing diaphragm atrophy. Our results not only contribute to our understanding of diaphragm abnormalities in ALS but also have potential importance in shaping the enrolment criteria for clinical trials specifically aimed at preserving or restoring diaphragm muscle mass or function (e.g. calcium channel sensitisers). In such trials, it is important to identify patients with advanced diaphragm atrophy because they are the most unlikely to respond to diaphragm-targeted interventions. In this view, the failure of tirasemtiv (a selective activator of the fast skeletal muscle troponin complex that increases its sensitivity to calcium) to meet the " 24 weeks supine FVC" primary endpoint of a randomised, placebo-controlled ALS trial $[42,43]$ could be due to the fact that the enroled patients had marked diaphragm atrophy in spite of a baseline supine FVC $\geqslant 70 \%$ predicted.

We suggest that a combination of sitting FRC, sitting IC and SNIP could be a more appropriate selection criterion than FVC for trials targeting the diaphragm, as a marker of slow-twitch diaphragm myofibre atrophy. Sitting IC could also be used for this purpose in routine practice. Recent advances in ultrasound evaluation of the diaphragm may also open up new avenues of research to identify better stratification criteria in diaphragm-targeted clinical trials in ALS [44, 45].

Acknowledgements: The authors wish to thank Marie-Laure Tanguy and Claudine Heisch-Pardon (Clinical Research Unit of the Pitié-Salpêtrière Hospital, Paris, France) for their help with data management for the study. We also would like to thank Maud Chapart (Myobank AIM, Pitié-Salpêtrière Hospital) for technical support, Gwenael Le Breton (AP-HP, Groupe Hospitalier Pitié-Salpêtrière Charles Foix, Paris, France) for her dedication to the study at the coordinating centre and Anne M. O'Rourke (O'Rourke Scientific and Medical Writing, San Diego, CA, USA) for editing the manuscript.

Author contributions: R. Guimarães-Costa, T. Similowski, J. Gonzalez-Bermejo and G. Bruneteau designed the study. R. Guimarães-Costa, M-C. Nierat, M.T. Bui, D. Akbar, J. Gonzalez-Bermejo and C. Morélot-Panzini contributed to data acquisition. R. Guimarães-Costa, T. Similowski, J. Gonzalez-Bermejo and G. Bruneteau performed the literature search. I. Rivals performed the statistical analysis. R. Guimarães-Costa, T. Similowski, J. Gonzalez-Bermejo and G. Bruneteau drafted the first version of the manuscript. All authors contributed to data interpretation, critically revised the manuscript for important intellectual content, approved the final version of the manuscript and agreed to be accountable for all aspects of the work.

The contributors to the RespiStimALS study were: Maria Del Mar Amador (Neurologist, Paris, France); Jean Christophe Antoine (Neurologist, St Etienne, France); Marie Christine Arne-Bes (Neurologist, Toulouse, France); Valérie Attali (Pneumologist, Paris, France); Katell Beauvais (Neurologist, Dijon, France); Veronique Brunaud-Danel (Neurologist, Lille, France); Gaelle Bruneteau (Neurologist, Paris, France); Jean-Philippe Camdessanche (Neurologist, St Etienne, France); William Camu (Neurologist, Montpellier, France); Laurence Carluer (Neurologist, Caen, France); Julien Cassereau (Neurologist, Angers, France); Maud Chapart (Pathologist, Paris, France); Bruno Chenuel (Neurologist, Nancy, France); Nathalie Chereau (Surgeon, Paris, France); Pascal Cintas (Neurologist, Toulouse, France); Pierre Clavelou (Neurologist, Clermont-Ferrand, France); Philippe Corcia (Neurologist, Tours, France); André Cornette (Pneumologist, Nancy, France); Philippe Couratier (Neurologist, Limoges, France); Isabelle Court-Fortune (Pneumologist, St Etienne, France); Antoine Cuvelier (Pneumologist, Rouen, France); Christophe Delclaux (Physiologist, Paris, France); Claude Desnuelle (Neurologist, Nice, France); Sophie Di Maria (Anaesthetist, Paris, France); Catherine Fargeot (Pharmacist, Paris, France); Brigitte Fauroux (Pneumologist, Paris, France); Armelle Finet-Monnier (Pneumologist, Marseille, France); Marie Celine Fleury (Neurologist, Strasbourg, France); Carmen Gaillat (Anaesthetist, Paris, France); Marjolaine Georges (Pneumologist, Dijon, France); Maurice Giroud (Neurologist, Dijon, France); Jesus Gonzalez-Bermejo (Pneumologist, Paris, France); Annick Greil (Pneumologist, Clermont Ferrand, France); Muriel Guerrier (Pharmacist, Paris, France); Nathalie Guy (Neurologist, Clermont Ferrand, France); Didier Hannequin (Neurologist, Rouen, France); Valérie Juillien (Pneumologist, Canne, France); Raul Juntas-Morales (Neurologist, Paris, France); Romain Kessler (Neurologist, Strasbourg, France); Yvan Kolev (Neurologist, Saint Brieuc, France); Julien Lagarde (Neurologist, Paris, France); Geraldine Lautrette (Neurologist, Limoges, France); Pierre Le Cam (Neurologist, Lyon, France); Nadine LeForestier (Neurologist, Paris, France); Gwendal Lemasson (Neurologist, Bordeaux, France); Thimothée Lenglet (Neurologist, Paris, France); Pascal Magro (Pneumologist, Tours, France); Anne Mallart (Pneumologist, Tours, France); Olivier Martinaud (Neurologist, Rouen, France); Vincent Meininger (Neurologist, Paris, France); Fabrice Menegaux (Surgeon, Paris, France); Nicole Meslier (Neurologist, Angers, France); Pierre Moirot (Pneumologist, Caen, France); Capucine Morelot-Panzini (Pneumologist, Paris, France); Yann Nadjar (Neurologist, Paris, France); Guillaume Nicolas (Neurologist, Angers, France); Myriam Niel-Duriez (Pneumologist, Paris, France); Marie-Cecile Nierat (Research coordinator, Paris, France); Severine Noullet (Surgeon, Paris, France); Nicolas Pageot (Neurologist, Paris, France); Thierry Perez (Pneumologist, Lille, France); Christophe Perrin (Pneumologist, Cannes, France), Odile Pillet (Neurologist, Bordeaux, France); Sophie Pittion (Neurologist, Nancy, France); Jean Pouget (Neurologist, Marseille, France); Pierre-François Pradat (Neurologist, Paris, France); Hélène Prigent (Pneumologist, 
Garches, France); Claudio Rabec (Pneumologist, Dijon, France); Jean-Damien Ricard (Pneumologist, Colombe, France); Norma Romero (Pathologist, Paris, France); Catherine Royer (Anaesthetist, Paris, France); François Salachas (Neurologist, Paris, France); Barbara Schaup (Anaesthetist, Paris, France); Kamila Sedkaoui (Pneumologist, Toulouse, France); Thomas Similowski (Pneumologist, Paris, France); Marie-Hélène Soriani (Neurologist, Nice, France); Christian Straus (Physiologist, Paris, France); Marie-Laure Tanguy (Statistician, Paris, France); Christine Tranchant (Physiotherapist, Nice, France); Nadia Vandenberghe (Neurologist, Lyon, France); Annie Vershueren (Neurologist, Marseille, France); Fausto Viader (Neurologist, Caen, France); Anne-Cecile Wienalek-Bachelet (Neurologist, Bordeaux, France).

Conflict of interest: R. Guimarães-Costa has nothing to disclose. T. Similowski reports personal fees from AstraZeneca, Boerhinger Ingelheim France, GSK Lungpacer Inc., TEVA, Chiesi, Pierre Fabre and Invacare, and personal fees and non-financial support from Novartis, outside the submitted work. In addition, he received honoraria from Synapse Biomedical to translate the DPS/NeurRx4 user manual from English into French in 2007 and, from 2012 to 2016, Synapse Biomedical contributed to a fundraiser organised by T. Similowski to promote respiratory research. T. Similowski is also engaged in scientific collaborations with two other companies manufacturing phrenic stimulation devices. With Lungpacer Inc (Canada) he acts as a paid consultant and investigator in clinical trials, while with Atrotech-Neuroresp (France) he acts as a probono consultant (no fees or other support). I. Rivals has nothing to disclose. C. Morélot-Panzini has nothing to disclose. M-C. Nierat has nothing to disclose M.T. Bui has nothing to disclose. D. Akbar has nothing to disclose. C. Straus has nothing to disclose. N.B. Romero has nothing to disclose. P.P. Michel has nothing to disclose. F. Menegaux has nothing to disclose. F. Salachas has nothing to disclose. J. Gonzalez-Bermejo has nothing to disclose. G. Bruneteau has nothing to disclose.

Support statement: RespiStimALS was funded by the Hospital Program for Clinical Research, French Ministry of Health (grant number P110133), a Contrat de Recherche Clinique of the Direction de la Recherche Clinique et du Développement, Assistance Publique-Hôpitaux de Paris, Paris, France (grant number CRC15017-R02), the French patients' association for ALS research (the Association pour la Rercherche sur la Sclérose Latérale Amyotrophique) and the Thierry de Latran Foundation for ALS. The study was also supported by the program Investissement d'Avenir ANR-10-AIHU 06 of the French Government. This work benefited from equipment and services provided by the CELIS cell culture platform (Institut du Cerveau et de la Moelle Epinière, Paris, France), a core facility supported through ANR-10-IAIHU-06 and ANR-11-INBS-0011-NeurATRIS funding.

\section{References}

1 Logroscino G, Traynor BJ, Hardiman O, et al. Descriptive epidemiology of amyotrophic lateral sclerosis: new evidence and unsolved issues. J Neurol Neurosurg Psychiatry 2008; 79: 6-11.

2 Polkey MI, Lyall RA, Yang $\mathrm{K}$, et al. Respiratory muscle strength as a predictive biomarker for survival in amyotrophic lateral sclerosis. Am J Respir Crit Care Med 2017; 195: 86-95.

3 Czaplinski A, Yen AA, Appel SH. Forced vital capacity (FVC) as an indicator of survival and disease progression in an ALS clinic population. J Neurol Neurosurg Psychiatry 2006; 77: 390-392.

4 Georges M, Golmard JL, Llontop C, et al. Initiation of non-invasive ventilation in amyotrophic lateral sclerosis and clinical practice guidelines: single-centre, retrospective, descriptive study in a national reference centre. Amyotroph Lateral Scler Frontotemporal Degener 2017; 18: 46-52.

5 Bourke SC, Bullock RE, Williams TL, et al. Noninvasive ventilation in ALS: indications and effect on quality of life. Neurology 2003; 61: 171-177.

6 Similowski T, Attali V, Bensimon G, et al. Diaphragmatic dysfunction and dyspnoea in amyotrophic lateral sclerosis. Eur Respir J 2000; 15: 332-337.

7 Arnulf I, Similowski T, Salachas F, et al. Sleep disorders and diaphragmatic function in patients with amyotrophic lateral sclerosis. Am J Respir Crit Care Med 2000; 161: 849-856.

8 Schiaffino S, Reggiani C. Molecular diversity of myofibrillar proteins: gene regulation and functional significance. Physiol Rev 1996; 76: 371-423.

9 Mizuno M. Human respiratory muscles: fibre morphology and capillary supply. Eur Respir J 1991; 4: 587-601.

10 Sanchez J, Medrano G, Debesse B, et al. Muscle fibre types in costal and crural diaphragm in normal men and in patients with moderate chronic respiratory disease. Bull Eur Physiopathol Respir 1985; 21: 351-356.

11 Gonzalez-Bermejo J, Morelot-Panzini C, Tanguy ML, et al. Early diaphragm pacing in patients with amyotrophic lateral sclerosis (RespiStimALS): a randomised controlled triple-blind trial. Lancet Neurol 2016; 15: 1217-1227.

12 Morelot-Panzini C, Niérat MC, Tanguy ML, et al. No benefit of diaphragm pacing in upper motor neuron dominant forms of amyotrophic lateral sclerosis. Am J Respir Crit Care Med 2018; 198: 964-968.

13 Brooks BR, Miller RG, Swash M, et al. El Escorial revisited: revised criteria for the diagnosis of amyotrophic lateral sclerosis. Amyotroph Lateral Scler Other Motor Neuron Disord 2000; 1: 293-299.

14 Cedarbaum JM, Stambler N, Malta E, et al. The ALSFRS-R: a revised ALS functional rating scale that incorporates assessments of respiratory function. J Neurol Sci 1999; 169: 13-21.

15 Noullet S, Romero N, Menegaux F, et al. A novel technique for diaphragm biopsies in human patients. J Surg Res 2015; 196: 395-398.

16 Engel WK. Selective and nonselective susceptibility of muscle fiber types. A new approach to human neuromuscular diseases. Arch Neurol 1970; 22: 97-117.

17 Jennekens FG, Tomlinson BE, Walton JN. Data on the distribution of fibre types in five human limb muscles. An autopsy study. J Neurol Sci 1971; 14: 245-257.

18 ATS/ERS statement on respiratory muscle testing. Am J Respir Crit Care Med 2002; 166: 518-624.

19 Morris CJ. Human skeletal muscle fibre type grouping and collateral re-innervation. J Neurol Neurosurg Psychiatry 1969; 32: 440-444

20 Buller AJ, Eccles JC, Eccles RM. Interactions between motoneurones and muscles in respect of the characteristic speeds of their responses. J Physiol (Lond) 1960; 150: 417-439.

21 Desypris G, Parry DJ. Relative efficacy of slow and fast alpha-motoneurons to reinnervate mouse soleus muscle. Am J Physiol 1990; 258: C62-C70. 
22 Bruneteau G, Simonet T, Bauche S, et al. Muscle histone deacetylase 4 upregulation in amyotrophic lateral sclerosis: potential role in reinnervation ability and disease progression. Brain 2013; 136: 2359-2368.

23 Hansen S, Ballantyne JP. A quantitative electrophysiological study of motor neurone disease. J Neurol Neurosurg Psychiatry 1978; 41: 773-783.

24 Frey D, Schneider C, Xu L, et al. Early and selective loss of neuromuscular synapse subtypes with low sprouting competence in motoneuron diseases. J Neurosci 2000; 20: 2534-2542.

25 Pun S, Santos AF, Saxena S, et al. Selective vulnerability and pruning of phasic motoneuron axons in motoneuron disease alleviated by CNTF. Nat Neurosci 2006; 9: 408-419.

26 Attali V, Mehiri S, Straus C, et al. Influence of neck muscles on mouth pressure response to cervical magnetic stimulation. Am J Respir Crit Care Med 1997; 156: 509-514.

27 Steier J, Kaul S, Seymour J, et al. The value of multiple tests of respiratory muscle strength. Thorax 2007; 62: 975-980.

28 Perry SF, Similowski T, Klein W, et al. The evolutionary origin of the mammalian diaphragm. Respir Physiol Neurobiol 2010; 171: 1-16.

29 Trumble DR, Duan C, Magovern JA. Effects of long-term stimulation on skeletal muscle phenotype expression and collagen/fibrillin distribution. Basic Appl Myol 2001; 11: 91-98.

30 Kovanen V, Suominen H, Heikkinen E. Mechanical properties of fast and slow skeletal muscle with special reference to collagen and endurance training. J Biomech 1984; 17: 725-735.

31 Patten BM, Zito G, Harati Y. Histologic findings in motor neuron disease. Relation to clinically determined activity, duration, and severity of disease. Arch Neurol 1979; 36: 560-564.

32 Froes MM, Kristmundsdottir F, Mahon M, et al. Muscle morphometry in motor neuron disease. Neuropathol Appl Neurobiol 1987; 13: 405-419.

33 Pinto S, Turkman A, Pinto A, et al. Predicting respiratory insufficiency in amyotrophic lateral sclerosis: the role of phrenic nerve studies. Clin Neurophysiol 2009; 120: 941-946.

34 Jenkins JA, Sakamuri S, Katz JS, et al. Phrenic nerve conduction studies as a biomarker of respiratory insufficiency in amyotrophic lateral sclerosis. Amyotroph Lateral Scler Frontotemporal Degener 2016; 17: 213-220.

35 Park JS, Park D. The terminal latency of the phrenic nerve correlates with respiratory symptoms in amyotrophic lateral sclerosis. Clin Neurophysiol 2017; 128: 1625-1628.

36 Holzbaur KR, Delp SL, Gold GE, et al. Moment-generating capacity of upper limb muscles in healthy adults. J Biomech 2007; 40: 2442-2449.

37 Oatis CA. Biomechanics of skeletal muscle. In: Kinesiology: the Mechanics and Pathomechanics of Human Movement. 2nd Edn. Philadelphia, Lippincott Williams \& Wilkins, 2009; pp. 45-68.

38 Brooke MH, Kaiser KK. Three "myosin adenosine triphosphatase" systems: the nature of their pH lability and sulfhydryl dependence. J Histochem Cytochem 1970; 18: 670-672.

39 Staron RS. Correlation between myofibrillar ATPase activity and myosin heavy chain composition in single human muscle fibers. Histochemistry 1991; 96: 21-24.

40 Perrie WT, Bumford SJ. Correlation of myosin heavy chains with ATPase staining of skeletal- and cardiac-muscle fibres. Biochem Soc Trans 1984; 12: 825-826.

41 Levine S, Nguyen T, Kaiser LR, et al. Human diaphragm remodeling associated with chronic obstructive pulmonary disease: clinical implications. Am J Respir Crit Care Med 2003; 168: 706-713.

42 Shefner J, Cudkowicz M, Hardiman O, et al. VITALITY-ALS: results of a phase III trial of tirasemtiv, a fast skeletal muscle troponin activator, as a potential treatment for patients with ALS. Amyotroph Lateral Scler Frontotemporal Degener 2017; 18: 16-17.

43 Andrews JA, Cudkowicz ME, Hardiman O, et al. VITALITY-ALS, a phase III trial of tirasemtiv, a selective fast skeletal muscle troponin activator, as a potential treatment for patients with amyotrophic lateral sclerosis: study design and baseline characteristics. Amyotroph Lateral Scler Frontotemporal Degener 2018; 19: 259-266.

44 Dube BP, Dres M, Mayaux J, et al. Ultrasound evaluation of diaphragm function in mechanically ventilated patients: comparison to phrenic stimulation and prognostic implications. Thorax 2017; 72: 811-818.

45 Oppersma E, Hatam N, Doorduin J, et al. Functional assessment of the diaphragm by speckle tracking ultrasound during inspiratory loading. J Appl Physiol 2017; 123: 1063-1070. 